\title{
An open prospective randomised trial to reduce the pain of blood glucose testing: ear versus thumb
}

\author{
Simon D Carley, Carole Libetta, Brian Flavin, John Butler, Nam Tong, Ian Sammy
}

Hope Hospital, Salford M6 8HD Simon D Carley specialist registrar in emergency medicine Carole Libetta specialist registrar in emergency medicine Brian Flavin specialist registrar in emergency medicine

John Butler specialist registrar in emergency medicine

Nam Tong clinical fellow in emergency medicine Ian Sammy consultant in emergency medicine Correspondence to: S D Carley, Department of

Emergency

Medicine,

Manchester Royal

Infirmary,

Manchester

M13 9WL

s.carley@

btinternet.com

BMJ 2000;321:20

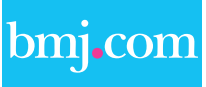

Further details about the method and an extra figure and table can be found on the BMJ's website
The assessment of random blood glucose concentration is one of the most commonly performed procedures in clinical practice. Traditionally, a digit is used-for accessibility and rich blood supply. The digits, however, are very sensitive, resulting in pain for some patients. Previous work showed that sampling from the side of the thumb was less painful than lancet sampling from the finger or from venepuncture at the elbow. ${ }^{1}$ During that study the earlobe was suggested as an alternative sample site, as it is also accessible and vascular. We tested the null hypothesis that there would be no difference in pain score between lancet skin puncture sites on the thumb and on the earlobe.

\section{Participants, methods, and results}

We conducted the study in a university hospital emergency department over three weeks; we received approval from the Salford and Trafford Health Authority's research ethics committee. We excluded patients aged under 16 years, and those who were dysphasic, less than alert on the AVPU ("Alert, responds to Voice, responds to Pain, Unresponsive") scale, ${ }^{2}$ had a bleeding disorder, or had altered sensation (including pain) or a lesion at any of the test sites.

We randomised eligible consenting patients to either earlobe or thumb sampling using a computer generated random number list (MS Excel 1997, Microsoft, Seattle, USA). We recruited 60 patients; 30 were assigned to each treatment group.

The skin was cleaned then wiped with a sterile dry swab to reduce potential interference with the sample. Skin puncture was performed on the lateral aspect of the thumb or on the earlobe by using the Unistik 2 device (Owen Mumford, Oxford). The area surrounding the test site was then squeezed to express a drop of blood. Once the blood was obtained, pain was assessed with a $100 \mathrm{~mm}$ visual analogue scale. ${ }^{3}$ The number of successful first attempts was recorded for each technique. If the prescribed method failed, the blood was taken from the lateral aspect of the thumb. Additional outcomes were adverse events and withdrawal from the trial.

Analysis was by intention to test. Mann-Whitney U tests were used for non-parametric data and $\chi^{2}$ tests with Yates's correction for categorical variables. SPSS for Windows, version 6 , was used for analysis. There were no statistical differences in terms of age, sex, experience of previous test, or diabetes between the groups (see the BMJ's website for a table of participants' characteristics and a chart showing the flow of participants through the study).

The median pain score was $2 \mathrm{~mm}$ in the ear group and $8.5 \mathrm{~mm}$ in the thumb group $(\mathrm{P}=0.01)$ (figure). There were five first time failures in the ear group compared with two in the thumb group $(\mathrm{P}=0.42)$.

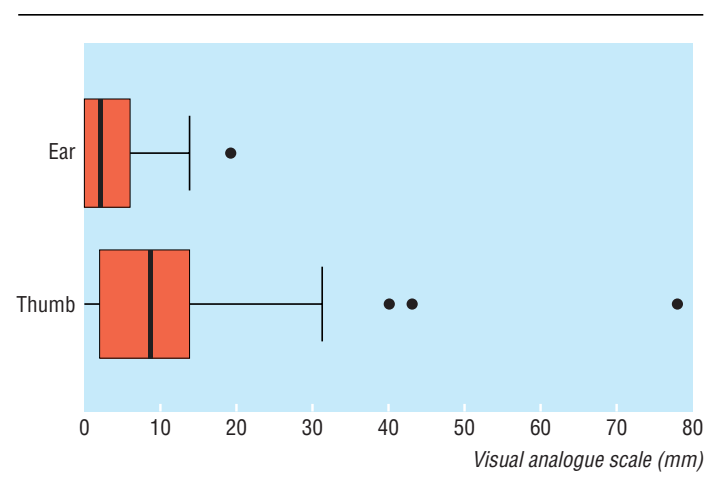

Distribution of pain scores. Dots represent the outliers, also included in the analysis. Vertical bands indicate medians, boxes 25th and 75th centiles, and whisker lines the largest values that are not outliers

There were no clinical adverse events and no withdrawals from the study.

\section{Comment}

Lancet skin puncture of the earlobe is less painful than that of the thumb. Although the difference in median pain scores is small, no patients in the ear group experienced high levels of pain. The reason for the difference in pain is unclear. The density of nociceptors may be lower in the ear than in the thumb, or the effect may be influenced by patients' perception, particularly as the patient cannot see the ear being tested.

The failure rate for both procedures was low, although it was slightly higher for sampling from the ear. This study was too small to detect a true difference if one exists.

The limitations of this study, particularly the lack of any data on repeat testing at the same site, will be apparent to those caring for patients who require frequent monitoring, and further research with these groups may be required.

Random blood glucose tests should be obtained by lancet puncture of the skin on the earlobe rather than by lancet puncture of the thumb.

Contributors: SDC initiated and supervised the study, analysed the data, and wrote the paper. IS, BF, CL, NT, and JB recruited patients and helped to write the paper. All authors participated in the design of the study. SDC is the guarantor of the study. Funding: None

Competing interests: None declared.

1 Loveland ME, Carley SD, Cranfield N, Hillier VF, Mackway-Jones K Assessment of the pain of blood-sugar testing: a randomised controlled trial. Lancet 1999;354:961.

2 Committee on Trauma. Advanced trauma life support program for physicians. 5th ed Chicago: American College of Surgeons, 1993.

3 Huskisson EC. Measurement of pain. Lancet 1974;ii:884-6.

(Accepted 5 April 2000) 